\title{
Pyridine-Containing Schiff Base Derivatives for the Structural Determination of Long-Chain Aldehydes by Gas Chromatography Combined with Mass Spectrometry
}

\author{
D. J. Harvey \\ Department of Pharmacology, University of Oxford, Oxford, United Kingdom
}

\begin{abstract}
Long-chain aldehydes, encountered as insect pheromones, were converted into Schiff bases with 3-aminopyridine, 3-(aminomethyl)pyridine, or 2-aminopyrimidine to provide derivatives suitable for revealing the alkyl chain structure by mass spectrometry. The two pyridine-containing derivatives were satisfactory in initiating a radical-induced cleavage of the chain to give a series of fragment ions, the masses and relative abundance of which revealed the chain structure. The derivatives were applied to aldehydes having straight, branched (iso and anteiso), and unsaturated (delta-7, delta-9, delta-11, and delta-13) structures; these all gave the fragmentation patterns that have been seen earlier for similar pyridine-containing derivatives of fatty acids (picolinyl esters) and alcohols (nicotinates). Of the two derivatives, those from 3-aminopyridine gave slightly simpler spectra. Derivatives formed from 2-aminopyrimidine were less satisfactory in revealing chain structure. (J $\mathrm{Am}$ Soc Mass Spectrom 1991, 2, 245-249)
\end{abstract}

$\mathrm{L}$ ong-chain aldehydes and ketones are frequently encountered as pheromones of both insects and lower vertebrates (see for example $[1,2])$. The alkyl chains of these compounds can have variuus structural features, such as unsaturation, that are difficult to determine by mass spectrometry without preparation of group-specific derivatives such as thiomethyl ethers of double bonds. Following the successful applications of pyridine-containing derivatives to the structural elucidation of fatty acids (picolinyl esters) [3-5], fatty alcohols (nicotinates) $[6,7]$, and glycerol ethers (nicotinylidine derivatives) [8], we extended this type of derivative to long-chain aldehydes by preparation of the Schiff base derivatives 1 , 2, and 3 from 3-aminopyridine, 3-(aminomethyl)pyridine, and 2-aminopyrimidine, respectively. Like the earlier pyridine-containing derivatives, these fragment under electron impact by charge localization on the pyridine residue and by abstraction of a hydrogen atom from the alkyl chain, to leave a radical site which then initiates a radical-site-induced fragmentation. This results in the spectrum containing a series of ions formed by cleavage at each carbon-carbon bond. The masses and relative abundances of these ions carry the required structural information. The advantage of derivatives of this type is that they

Address reprint requests to D. J. Harvey, Department of Pharmacology, South Parks Road, Oxford, OX1 3QT, UK. involve derivatization of the functional group of the compound rather than the structural features of the alkyl chain, such as unsaturation or branching, and are thus applicable to all chain types. This article describes derivatives of this type applicable to longchain aldehydes.

\section{Experimental}

\section{Materials}

Straight-chain primary alcohols $\left(C_{10}, C_{11}, C_{12}, C_{14}\right.$, $C_{16}$, and $C_{18}$ ), cis-7-tetradecenal, cis-9-hexadecenal, cis-11-hexadecenal, cis-13-octadecenal, and N,O-bis(trimethylsilyl)-trifluoroacetamide (BSTFA) were obtained from Sigma Chemical Co. (Poole, Dorset, UK). 3-Aminopyridine, 3(aminomethyl)pyridine, and nonanal were from Aldrich Chemical Co. (Gillingham, Dorset, UK). Branched-chain fatty acids (isoand anteiso- $\mathrm{C}_{14}-\mathrm{C}_{21}$ ) were obtained from Applied Science Laboratories (State College, PA) and were reduced to the corresponding alcohols with lithium aluminum hydride in ether. 2-Aminopyrimidine was obtained from the Dyson Perrins Laboratory (Oxford, UK). The straight-chain and branched-chain aldehydes were synthesized from the corresponding alcohols by oxidation with chromium trioxide in pyridine for $30 \mathrm{~min}$ at room temperature followed by solvent (ethyl acetate) extraction. 
<smiles>[R]C=Nc1ncccn1</smiles>

Structure 3

\section{Schiff Base Derivatives of Long-Chain Aldehydes}

The aldehyde (about $0.1 \mathrm{mg}$ ) was dissolved in acetonitrile and heated for $15 \mathrm{~min}$ with about $1 \mathrm{mg}$ of either 3-(aminomethyl)pyridine, 3-aminopyridine, or 2aminopyrimidine. Aliquots of these solutions were injected directly into the gas chromatography combined with mass spectrometry (GC/MS) instrument.

\section{Gas Chromatography Combined with Mass Spectrometry}

Gas chromatography combined with mass spectrometry data were recorded with a VG 70/70F mass spectrometer, (Manchester, UK) interfaced to a Varian 2440 gas chromatograph (Palo Alto, CA) that was fitted with an SGE (Ringwood, Australia) split-splitless injection system operated in the split mode with a split ratio of 10:1. The column was a $25 \mathrm{~m} \times 0.2 \mathrm{~mm}$ OV-1 bonded-phase fused-silica capillary, film thickness $0.33 \mu \mathrm{m}$ terminating $10 \mathrm{~mm}$ inside the mass spectrometer ion source. Helium at $1 \mathrm{~mL} / \mathrm{min}$ (measured in the absence of the mass spectrometer vacuum) was used as the carrier gas. Operating conditions were as follows: injector, transfer line, and ion source temperatures, 300,300 , and $280^{\circ} \mathrm{C}$, respectively, column oven temperature programmed in the range 200-350 ${ }^{\circ} \mathrm{C}$ at $4{ }^{\circ} \mathrm{C} / \mathrm{min}$ to give a retention time of about $5 \mathrm{~min}$ for each sample; accelerating voltage, $4 \mathrm{kV}$; electron energy, 70 and $25 \mathrm{eV}$; trap current, $1.0 \mathrm{~mA}$; scan speed, $1 \mathrm{sec} /$ decade. Spectra were acquired and processed with a VG $11 / 250$ data system.

\section{Results and Discussion}

\section{Derivatives with 3-Aminopyridine}

These compounds formed in near quantitative yield and were examined by GC/MS by direct injection of the reaction mixture into the chromatograph. Gas liquid chromatography properties were generally good with little tailing. To demonstrate stability towards trimethysilylating reagents, an aliquot was blown to dryness, dissolved in BSTFA, and a sample was injected. This gave the same result as before. The reaction worked equally well at the low microgram level and was thus suitable for use with biological samples.

Straight-chain aldehydes. The $25-\mathrm{eV}$ electron impact mass spectrum of the 3-aminopyridine Schiff base derivative of $n$-octadecanal is shown in Figure 1 . The expected radical-induced fragmentation gives a series of ions separated by $14 \mathrm{u}$ (see Scheme I), but the relative abundance of these was not as great as in the corresponding spectra of picolinyl [3-5] or nicotinate $[6,7]$ derivatives of acids and alcohols, respectively (note the $\times 10$ multiplication factor above mass 140 in Figure 1). Use of $25 \mathrm{eV}$ rather than $70 \mathrm{eV}$ reduced the relative abundance of the low mass ions by a factor of 2-3, but there was no significant loss in sensitivity. The molecular ion, on the other hand, was satisfactorily abundant. The base peak at $m / z 120$ appeared to have been formed by hydrogen migration to the pyridine moiety with cleavage to give the allylic radical ion $b$ (Scheme II). This was supported by corresponding base peaks in the spectra of the other Schiff base derivatives discussed below; the source of the migrating hydrogen atom was not determined. The high relative abundance of the ion at $\mathrm{m} / \mathrm{z} 133$ can be accounted for by formation of the conjugated ion $c$ (Scheme III). From here to the $[\mathrm{M}-1]^{+}$ion at $m / z$
Figure 1. Electron impact mass spectrum $(25 \mathrm{eV})$ of the 3-aminopyridine Schiff base derivative of $n$-octadecanal.

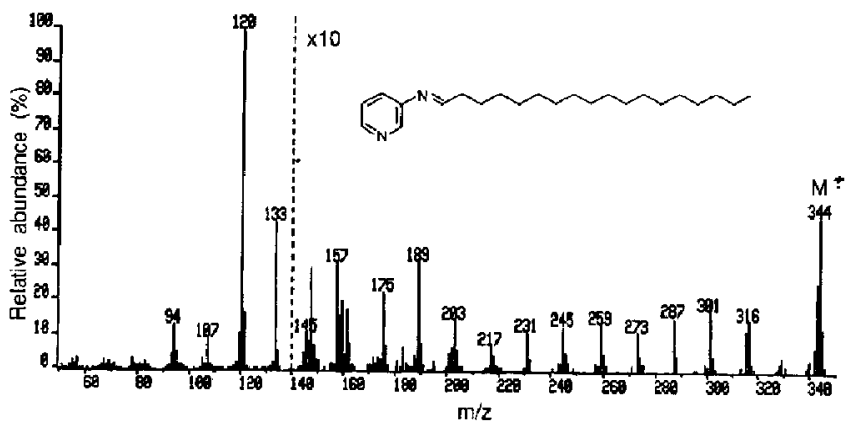




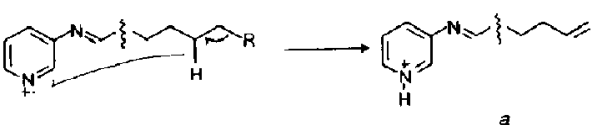

Scheme I

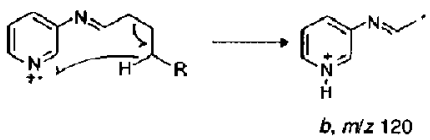

Scheme II

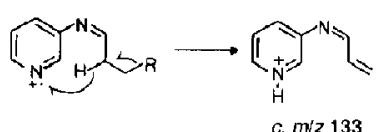

Scheme III

343 , the diagnostic series of ions is $m / z 147,161,175$, $189,203,217,231,245,259,273,287,301,315$, and 329. The structures of the two ions at $\mathrm{m} / z 157$ and 159 are unclear, although they appeared to contain the pyridine ring.

Branched-chain aldehydes. The spectrum of the 3aminopyridine Schiff base derivative of iso-octadecanal (16-methyl-heptadecanal) is shown in Figure 2. This shows the same general features as the spectrum of the straight-chain compound discussed above, with the expected change in the pattern of ions revealing the position of the branchpoint [3]. This structural feature is apparent by (1) the low abundance of the ion at $m / z 315$, reflecting the fact that the molecule cannot lose $29 \mathrm{u}$ from the end distal to the pyridine ring and (2) the relatively high abundance of the adjacent ions at $m / z \quad 301$ and 329 caused by cleavage at the tertiary carbon atom. In this respect the fragmentation is strictly analogous to that of the picolinyl and nicotinate derivatives discussed earlier. Anteisoaldehydes showed similar behavior, with the missing ion corresponding to $[\mathrm{M}-43]^{+}$(Figure 3).

Unsaturated aldehydes. The spectrum of cis-13-octadecenal is shown in Figure 4 . The ions in the lower half of the spectrum were the same as those in the spectra of the saturated compounds just discussed, but those in the upper half of the spectrum showed the pattern expected for monounsaturation [3]. Thus, the molecular ion was abundant, and the 40 rather than $42 \mathrm{u}$ difference between the ions $d \mathrm{~m} / \mathrm{z} 245$ and $f \mathrm{~m} / \mathrm{z} 285$ located the position of unsaturation. The relatively high abundance of the characteristic ions at $m / z 245(d), 299(g)$, and $313(h)$ can be accounted for by the mechanisms shown in Schemes IV-VI, respectively. Ion $d$ involves the loss of an allylic radical, whereas ions $j$ and $h$ can be rationalized as involving abstraction of one of the allylic hydrogen atoms. The spectra of the homologous unsaturated aldehydes (see Materials) gave similarly diagnostic spectra but with a drop in the relative abundance of ion $g$. It has been observed $[9,10]$ with the spectra of the picolinyl derivatives of the fatty acids that unsaturation closer to the oxygenated function than $\mathrm{C}-7$ results in a loss in the elevated relative abundance of this ion although the increased abundance of ion $h$ remains unchanged.

\section{Derivatives with 3-(Aminomethyl)pyridine}

The spectra of these derivatives were strictly analogous to those of the 3-aminopyridine derivatives just discussed (see Figure 5) with the addition of several relatively abundant ions in the lower half of the spectrum. These were produced as shown in Scheme VII. The shift in the base peak from $m / z 120$ in the spectra of the 3-aminopyridine derivatives to $m / z 134$ in the spectra of these 3-(aminomethyl)pyridine Schiff bases supports the structure for $m / z 120$ proposed above. The ion at mass 203 corresponds to that at $m / z 189$ in the above spectra.

\section{Derivatives with 2-Aminopyrimidine}

The 2-aminopyrimidine group did not abstract a hydrogen atom from the aliphatic chain as effectively as the pyridine ring and, consequently, although the spectra showed the normal series of radical-induced ions, these were relatively weak and did not adequately reflect the position of methyl branch points as can be seen in Figure 6. However, the molecular ions were abundant. Ions of the type discussed above,

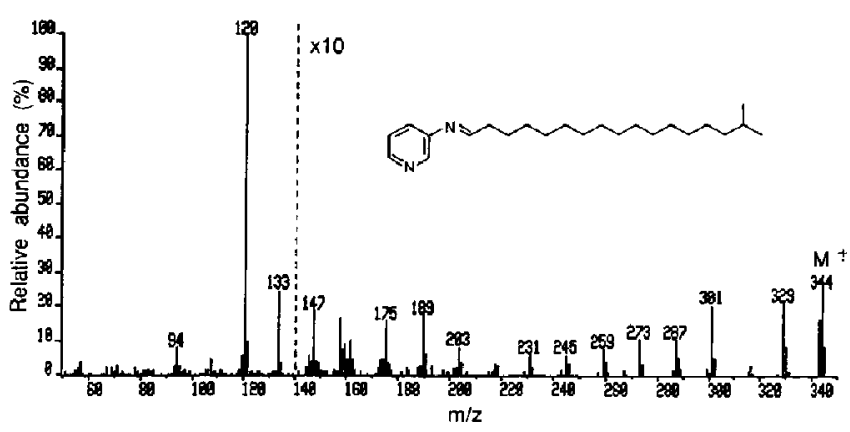

Figure 2. Electron impact mass spectrum (25 eV) of the 3-aminopyridine Schiff base derivative of isooctadecanal. 
Finare 3. Electron impact mass spectrum (25 eV) of the 3-aminopyridine Schiff base derivative of anteise-hereicosanal.
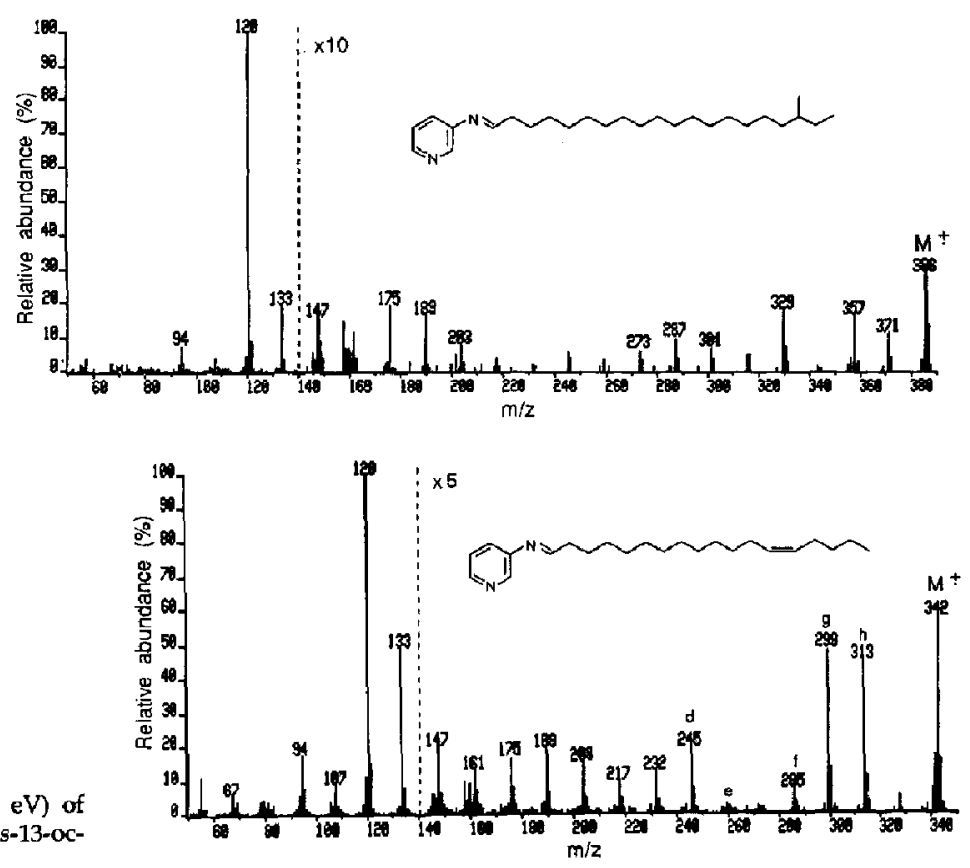

Figure 4. Electron impact mass spectrum $(25 \mathrm{eV})$ of the 3-aminopyridine Schiff base derivative of cis-13-octadecenal.

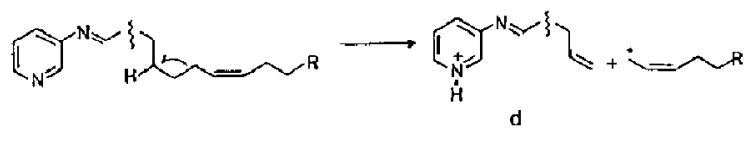

Scheme IV

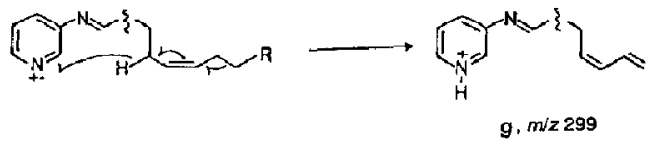

Scheme V

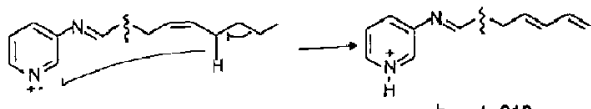

Scheme VI

Figure 5. Electron impact mass spectrum $(25 \mathrm{eV})$ of the 3-(aminomethyl)pyridine Schiff base derivative of cis-13octadecenal.

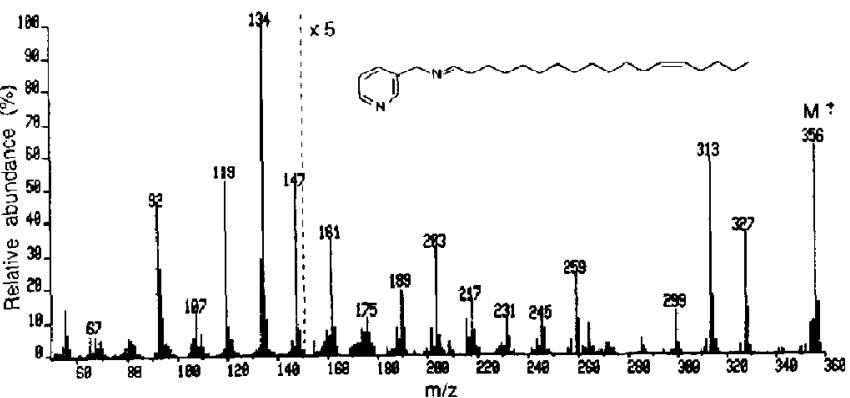




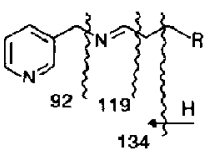

Scheme VII

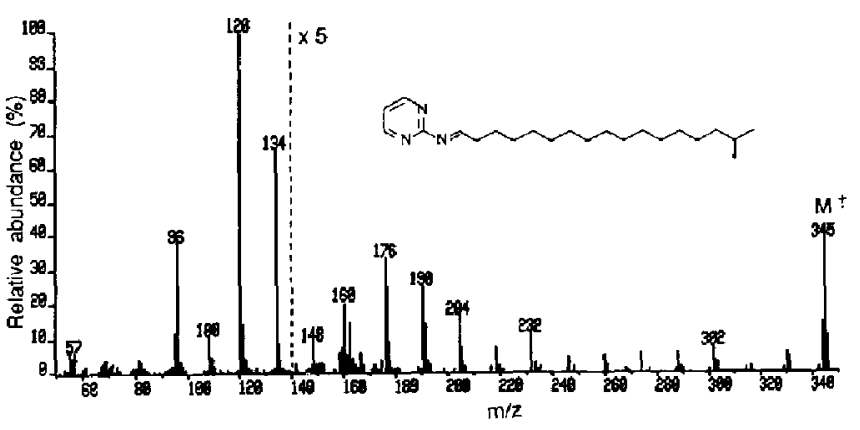

Figure 6. Electron impact mass spectrum $(25 \mathrm{eV})$ of the 2-aminopyrimidine Schiff base derivative of isoeicosanal. reflecting unsaturation, were present in the spectra of unsaturated aldehydes, but were relatively weaker than those in the spectra of the other Schiff base derivatives. Therefore, these derivatives were not investigated further. Ions in the lower part of the spectrum reflect predictable cleavages adjacent to the pyrimidine ring.

\section{Conclusions}

In conclusion, Schiff base derivatives from both 3aminopyridine and 3-(aminomethyl)pyridine were suitable for revealing the structure of straight-, branched- and unsaturated-long-chain aldehydes by initiating radical-induced cleavages of the alkyl chain in a manner similar to that observed previously for analogous derivatives of long-chain acids and alcohols. Although both derivatives gave fragment ions of comparable relative abundance, the simpler spectra produced by the 3-aminopyridine Schiff bases made them marginally better as analytical derivatives. Because the fragmentation of these compounds was found to be strictly analogous to that displayed by corresponding pyridine-containing derivatives of fatty acids and alcohols, it would be expected that other alkyl chain structures, such as cyclopropane rings, could be similarly identified.

\section{Acknowledgments}

The mass spectroneter was originally purchased with the aid of a grant from the Medical Research Council. Funds were also provided by the Wellcome Trust.

\section{References}

1. Brand, J. M.; Young, J. C.; Silverstein, R. M. Fortschr. Chem. Org. Naturst. 1979, 37, 1-190.

2. Aldrich, J. R.; Lusby, W. R.; Kochansky, P. Experientia 1986, 42, 583-585.

3. Harvey, D. J. Biomed. Mass Spectrom. 1982, 9, 33-38.

4. Harvey, D. J. Biomed. Mass Spectrom. 1984, 11, 187-192.

5. Harvey, D. J. Biomed. Mass Spectrom. 1984, 11, 340-347.

6. Vetter, W.; Meister, W. Org. Mass Spectrom. 1981, 16, 118-122.

7. Harvey, D. J.; Tiffany, J. M. Biomed. Mass Spectrom. 1984, $11,353-359$.

8. Harvey, D. J. Biomed. Environ. Mass Spectrom. in press.

9. Christie, W. W.; Brechany, E. Y.; Holman, R. T. Lipids 1987, 22, 224-228.

10. Harvey, D. J. Biomed. Environ. Mass Spectrom. 1989, 18, 719-723. 